\title{
Spermienfunktion - gestern, heute und morgen ...
}

\author{
E. Ernst, W. Weidner \\ Urologische Klinik der Universitätsklinik Gießen
}

Am 18./19. November 1994 fand in Heidelberg das Andrologie-Symposion Spermienfunktion gestern, heute und morgen statt, an dem über 120 andrologisch intressierte Reproduktionsmediziner, Urologen, Dermatologen und Reproduktionsbiologen teilnahmen.

Das Symposion, das von Prof. Runnebaum, dem gynäkologischen Endokrinologen der Univ. Frauenklinik Heidelberg ausgerichtet wurde, erhob den Anspruch, aus reproduktionsmedizinischer Sicht Andrologie zum Zeitpunkt 1994 in Basisforschung und Klinik darzustellen.

\section{Grundlagenforschung}

Skakkebaek (Kopenhagen/Dänemark) berichtete erneut, daß er eine Abnahme von Spermatozoenzahl und Qualität im Laufe der letzten Jahrzehnte beobachtet habe. Diese Daten seines Kopenhagener Labors wurden kontrovers diskutiert; Kernpunkte der Kritik waren, daß es sich um wechselnde Untersucher, unterschiedliche Qualitätskontrollen und wechselnde Karenzzeiten im zeitlichen Verlauf der Untersuchung handelte.

Vogt (Heidelberg) berichtete in seinem sehr interessanten Referat über moderne genetische Aspekte der männlichen Fertilität unter besonderer Berücksichtigung neuerer reproduktionsmedizinischer Techniken. Derzeit werden 615 Gene mit einer gestörten Reproduktion in Zusammenhang gebracht. Klinische Relevanz hat u.a. das CF-Gen (CFTR) erreicht, das für die Ausbildung einer zystischen Fibrose und der bilateralen kongenitalen Duktusaplasie (CBAVD) wichtig ist. In diesem Zusammenhang werden mindestens 10 bekannte Mutationsmöglichkeiten diskutiert, wobei die Wahrscheinlichkeit, ein phänotypisch krankes Kind zu bekommen, nur dann sehr hoch ist, wenn beide Partner Träger einer CF-Abnormalität sind. Wenn die Frau keine Trägerin ist, besteht ein geringes Risiko. Darum ist es vor einer entsprechenden mikrochirurgischen Operation, d.h. epididymaler Spermatozoenaspiration und IVF der Partnerin mit ICSI, sinnvoll, den Vater und die Mutter genetisch zu untersuchen. Ein weiterer wichtiger Aspekt ist der Azoospermie-Faktor, der auf dem YChromosom lokalisiert wird. Wenn aufgrund reproduktionsmedizinischer Therapie ein solcher Patient Vater von Söhnen wird, so sind $100 \%$ dieser Söhne azoosperm. In der Diskussion wurde die Frage aufgeworfen, wie vorgegangen werden soll, wenn Patienten nach der genetischen Analyse ein hohes Risiko eingehen würden, evtl. ein an Mukoviszidose erkranktes Kind zu bekommen. Hier konnte kein Konsens gefunden werden, wobei darauf hingewiesen

Akt. Urol. 26 (1995) 216-218

(C) Georg Thieme Verlag Stuttgart · New York wurde, daß in den meisten Fällen (über 90\%) Embryonen mit genetischen Veränderungen abortiert werden.

Frau Töpfer-Petersen (Hannover) und Overstreet (Davis, USA) diskutierten neue molekulare Erkenntnisse der Spermatozoen-Oozyten Interaktion bzw. deren Beeinflussung durch die Sekretion des weiblichen Genitaltraktes bei der Befruchtung. Die Verschmelzung des Spermatozoons mit der Eizelle setzt bekanntlich die primäre Bindung an die Zona pellucida voraus. Diese setzt die akrosomale Reaktion in Gang, die ihrerseits die sekundäre Bindung des Spermatozoons und die proteolytische Zerstörung der Zona initiiert. Erst dann kommt es zur Bindung an die Zellmembran und zum Eindringen des Spermatozoons in das Ei. Wichtig für die erste Bindung sind Kohlenhydratbindungsproteine an der Spermatozoenaußenfläche, z. B. am Äquatorialsegment. Diese werden schon im Rete testis gebildet. Die Inhibitoren aus dem Seminalplasma verhindern die verfrühte Bindung und werden vor der Kapazitation entfernt. Die Passage der Spermatozoen durch den weiblichen Genitaltrakt ist zum einen sehr schnell, zum anderen aber auch langsam migrierend möglich. Im Tierexperiment konnte gezeigt werden, daß einige Spermatozoen in Minuten, andere erst innerhalb von Stunden, das Ei erreichen. Dieser Migrationsproze $ß$ wird durch Muskelkontraktionen der Zervix unterstützt, die chemisch oder physikalisch von den Oozyten getriggert werden. Ein Großteil der Spermatozoen verbleibt jedoch einige Zeit in der Zervix, die damit eine Reservoirfunktion erfüllt. Der Mukus selbst ist nur unter hormoneller Beeinflussung um den Zeitraum der Ovulation für die Spermatozoen durchgängig und hat eine gute Filterfunktion. Erst wenn das Spermatozoon den Mukus verlassen hat, wird die akrosomale Reaktion gestartet.

\section{Klinische Diagnostik}

Diese Sitzungsreihe wurde durch die reproduktionsmedizinische Ausrichtung der Veranstaltung geprägt.

Schulze (Hamburg) gab einen Überblick über die notwendige klinisch-andrologische Diagnostik unter besonderer Berücksichtigung der Indikation zur Hodenbiopsie. Er wies darauf hin, daß seines Erachtens die hypergonadotrope Azoospermie als Indikation zur Hodenbiopsie gilt, um die heute diskutierten verschiedenen Formen des Sertoli-Cell-only-Syndroms, frühzeitig zu erfassen. Er wies weiter darauf hin, daß nach seiner Meinung die Indikation zur elektronenmikroskopischen Spermatozoenanalyse relativ weit gestellt werden sollte, da diese Methode in ihrer Reproduzierbarkeit und Aufdeckung ultrastruktureller Defekte bei reduzierter Motilität gegenüber normalen morphologischen Verfahren große Vorteile bietet. 
Menkveld (Tygerberg/Südafrika) sprach über die Vorteile der Tygerberg-Klassifikation zur morphologischen Differenzierung von Spermatozoen. Er wies darauf hin, daß humanes Sperma als morphologisch sehr heterogen anzusehen ist. Die Vorteile der strikten morphologischen Kriterien der Tygerberg-Klassifikation liegen seines Erachtens in der besonderen Reproduzierbarkeit der Ergebnisse und der besseren Assoziation zur Fertilisierungsrate unter In-vitro-Fertilisations-Bedingungen.

Krause (Marburg) sprach über den heutigen Stand der computerisierten Spermatozoenanalyse (CASA). Die Vorteile der Methode liegen in der Objektivierbarkeit und Präzisierung der Ejakulatbefundung, während die biologische Aussagefähigkeit bisher nicht verbessert werden kann. Bei der Motilitätsbestimmung sind die Vorteile groß, da die Überschätzung der Motilität bei wenig motilen Spermatozoen und die Unterschätzung bei sehr vielen motilen Spermatozoen in der Routineauswertung verhindert werden kann. Die Motilität wird im Schnitt mit der automatisierten Analyse in etwa $10 \%$ geringer angegeben als in der Zählkammer. Unter Verwendung neuer Softwareprogramme ist auch versucht worden, die Morphologie im Rahmen einer automatisierten Analyse zu erfassen. Die Ergebnisse sind jedoch bisher nicht befriedigend. In der Diskussion wurde angemerkt, $\mathrm{da} \beta$ es bisher keine Standards für die automatisierte Spermatozoenanalyse gäbe. Es sei bisher auch nicht gelungen, pathologische Bewegungsmuster für die Motilitätsanalyse der Spermatozoen zu erarbeiten, was ein großes Manko sei. Daraus resultierte die Forderung, kurzfristig Standards zumindest im deutschsprachigen Raum für die Benutzer entsprechender Untersuchungssysteme zu erarbeiten.

Frau Eggert-Kruse (Heidelberg) berichtete über die immunologischen Aspekte der männlichen Subfertilität. SPAK (Spermatozoenantikörper) haben bei Nachweis im Serum keine Bedeutung, ihre Bestimmung ist obsolet. Lokale Spermatozoenantikörper, wie sie z.B. durch den MAR- oder den Immunobead-Test nachgewiesen werden, sind sehr selten (IgG 1,6\%, IgA $1 \%$ ) und haben, wenn sie vorkommen, wahrscheinlich einen negativen Einfluß auf die Fertilität. Der Postkoitaltest wird dann häufig negativ. Bei einem Nachweis-Titer von IgA von über $30 \%$ (im MAR-Test) konnten keine Schwangerschaften mehr nachgewiesen werden.

Van Kooij (Utrecht), Hartmann (Heidelberg) und Kremer (Groningen) sprachen anschließend über verschiedene Spermatozoenfunktionsteste. Es bestand Einigkeit, da $\beta$ der Hamsteroozytentest derzeit kein klinisch relevantes Verfahren zur Beurteilung männlicher Fertilität sei. Der Nachweis akrosomaler Reaktionen erfolgt entweder durch die Gelatinolyse oder mittels Tripel-Stain-Technik, Immunfluoreszens oder monoklonale Antikörper. Bekanntlicherweise fehlt Rundkopfspermien die akrosomale Reaktion, vermindert ist die akrosomale Reaktion auch bei Teratozoospermie, OAT-Syndrom, Polyzoospermie und Dekapitationssyndrom. Es besteht ein eindeutiger Zusammenhang zwischen geringer akrosomaler Reaktion, Einschränkung der Spermatozoenqualität und schlechter Fertilisierungsrate bei IVF. Einschränkend wurde darüber be- richtet, daß zumindest im Tierreich bei transgenen Mäusen, denen das Akrosom fehlt, Fertilisierungen beobachtet worden sind.

Kremer (Groningen) empfahl noch einmal dringend die Durchführung von Sperma-Mukus-Interaktionstesten bei allen reproduktionsmedizinischen Fragestellungen: den Postkoitaltest, den Spermien-PenetrationsMeter-Test und den Spermien-Zervikal-Mukus-KontaktTest. Die 3 Verfahren werden nicht gleichsinnig angewendet; weitgehend Einigkeit bei den Reproduktionsmedizinern bestand jedoch darin, grundsätzlich einen Postkoitaltest mit Ablesung nach 12 Stunden durchzuführen.

\section{Therapeutische Aspekte}

Schill (Gießen) und Nieschlag (Münster) gaben einen Überblick über den derzeitigen Stand der medikamentösen Therapie in der Andrologie. Übereinstimmung bestand darin, daß eine Therapie des idiopathischen OAT-Syndroms medikamentös nicht möglich ist. Grundsätzlich ist eine kausale Therapie anzustreben, wie z. B. bei der Spermatozoentransportstörung oder dem Hypogonadismus. Dies gilt auch für die antibiotische und antiphlogistische Therapie der urogenitalen Infektion. Ob Aromatasehemmer und Mastzellblocker, die in vitro wirksam sind, klinisch eingesetzt werden können, ist umstritten. 9 Prozent der Subfertilität beim Mann ist nach Münsteraner Ergebnissen endokrin bedingt. Hier hat die medikamentöse Therapie (GnRH, HCG/HMG, Testosteron) in der Behandlung des hypogonadotropen Hypogonadismus ihre Berechtigung. Leider haben sich die ersten positiven Erfahrungen, die diese Substanzen zur Behandlung des OAT-Syndroms empfohlen haben, nicht bestätigen lassen. Eine Doppel-Blindstudie mit HCG/HMG gegen Plazebo konnte bei dieser Indikation keinen Effekt zeigen, Auch das Einsetzen einer Zykomatpumpe zeigte keine Verbesserung der Spermatozoenqualität beim OAT-Syndrom.

Weidner (Gießen) faßte die derzeitigen Ergebnisse bezüglich Durchgängigkeit und Schwangerschaftsraten bei der operativen Therapie der Verschlußazoospermie zusammen. Die mikrochirurgische Vasovasostomie und Epididymovasostomie ist Standard, MESA (Micro-Epididymal-Sperm-Aspiration) und TESE (Testikuläre-Sperm-Exzision) sowie evtl. in Zukunft erneut die alloplastische Spermatozele sollten immer in Kombination mit einer Mikromanipulation (ICSI) durchgeführt werden.

Über die Therapie der Varikozele berichteten Comhaire (Ghent, Belgien) und Nieschlag (Münster). Nach Meinung von Comhaire ist die Varikozele mit der Abnahme des Hodenvolumens auf der betroffenen Seite und der Spermatozoenqualität, die um so schlechter wird, je länger die Varikozele besteht, assoziiert. Außerdem hat die Varikozele einen negativen Einfluß auf die Sertoli- und die Leydigzellfunktion mit Abnahme des Plasmatestosteronwertes. Es bestand Einigkeit, eine Therapie der Varikozele bei schwerem OAT-Syndrom mit erhöhtem FSH-Wert und erniedrigtem Hodenvolumen nicht durchzuführen. Nach Meinung von Comhaire zeigt die derzeit laufende WHOStudie, daß die Therapie der Varikozele die Fertilitätschancen des infertilen Mannes verbessert. Völlig konträr sieht dies Nieschlag. Seine prospektive Studie hat gezeigt, 
daß die Therapie der Varikozele bei andrologischem Sterilitätsfaktor im Vergleich mit einem "wait and see" zu keiner erhöhten Schwangerschaftsrate in der Partnerschaft führt. Er meint, daß es dringend notwendig ist, die Patienten noch besser zu selektionieren, die von einer derartigen Therapie profitieren. In der Diskussion wurde eindeutig festgehalten, daß eine präventive Operation, ebenso wie die Therapie der subklinischen Varikozele, abgelehnt werden muß. Kontrovers wurde die neue Technik der antegraden Sklerosierung diskutiert, wobei Comhaire von der antegraden Varikozelensklerosierung als rein experimentellem Verfahren sprach, das seinen Wert in prospektiv-vergleichenden Studien bisher nicht bewiesen hat.

Te Velde (Utrecht/Holland) sprach über die Wirksamkeit einer intrauterinen Insemination (IUI) bei andrologischem Sterilitätsfaktor. Unbestritten ist die Indikation beim zervikalen Sterilitätsfaktor. Bei leichter männlicher Subfertilität sind nach seiner Meinung mit einer milden ovariellen Hypostimulation der Partnerin die intrauterinen Inseminationsergebnisse $\mathrm{zu}$ verbessern. Insgesamt liegen die Schwangerschaftsraten bei ca. $10 \%$. Ob die IUI bei einem schweren andrologischen Sterilitätsfaktor überhaupt einen Nutzen hat, wurde kritisch diskutiert. Comhaire erklärte dazu, daß nach seinen Erfahrungen die Aufarbeitung der Spermatozoen für die intrauterine Insemination ganz entscheidend sei. Die Percoll-aufgearbeiteten Spermatozoen sollen eine höhere SS-Rate per Zyklus zeigen. Diedrich (Lübeck) sprach über die Erfolge seiner Arbeitsgruppe mittels IVF in bezug auf die andrologisch bedingten Sterilitätsfaktoren, die $30 \%$ aller Indikationen zur IVF ausmachen. Nach Selektionierung qualitativ guter Spermatozoen, z. B. durch swim-up, erreicht er mit der IUI nur Schwangerschaftsraten von $4-8,8 \%$ pro Zyklus. Mit der IVF mit anschließendem Embryonentransfer berichtet seine Arbeitsgruppe über SS-Raten von 14-17\%/Zyklus und Geburtsraten von 10-15\%/Zyklus. Er empfiehlt nach erfolgloser Insemination (3-6 Zyklen) immer die Durchführung einer IVF zur Therapie des andrologischen Sterilitätsfaktors. Bei erfolgloser Standard-IVF kommt seines Erachtens dann der Einsatz einer Mikromanipulationstechnik, z. B. ICSI in Frage. Michelmann (Göttingen) faßte diese beiden Referate noch einmal zusammen und gab $\mathrm{Mi}$ nimalkriterien der Spermienqualität beim andrologischen Faktor zur IUI und IVF an. Schlechte Fertilisierungschancen liegen nach seiner Meinung bei Spermatozoen-Konzentrationen von $<2 \mathrm{Mill} / \mathrm{ml}$, Motilität von $<10 \%$, Progressiv-Motilität von $<25 \%$ und normaler Morphologie von $<5 \%$ vor. Abgeschlossen wurde dieser therapeutische Block durch ein Referat über die Entwicklungen in der Brüsseler Arbeitsgruppe durch die Einführung von ICSI in die Reproduktionsmedizin. Devroey (Brüssel) gab an, der Erfolg von ICSI hänge weder von der Zahl der Spermatozoen noch von der Morphologie ab. Einzig wichtig sei, motile Spermatozoen zu benutzen, da dies ein Vitalitätskriterium sei. Die neuesten Ergebnisse der Brüsseler Arbeitsgruppe haben gezeigt, daß auch kryokonservierte Nebenhoden- und Hodenspermatozoen die gleiche Fertilisierungschancen aufweisen wie frisch aspirierte Spermatozoen. Dies eröffnet neue zeitliche und räumliche Dimensionen für die Kooperation zwischen Mikrochirurgen und Reproduktionsmedizinern. Mittels TESE und ICSI konnten inzwischen Fertilisierungsraten von $57 \%$ und Schwangerschaftsraten von $31 \%$ erreicht werden. Darüber hinaus wies Devroey darauf hin, daß auch bei nicht-obstruktiver Azoospermie mit normalem FSH in 99,9\% Spermatozoen aus dem Hoden durch ausgiebige Hodengewebeexzision gewonnen werden könnten. Dies würde zu einer neuen Ära in der Therapie der nicht-obstruktiven Azoospermie führen. Es wurde diskutiert, ob in diesen Fällen präoperativ eine Hodenbiopsie zur Objektivierung der Spermatogenese durchgeführt werden sollte. Dies wurde abgelehnt, um eine Schädigung des Hodens mit Reduzierung der Möglichkeiten einer erneuten Gewebsentnahme zu vermeiden. Edwards (Cambridge) wies abschließend darauf hin, daß nur $60 \%$ der Eizellen fertilisiert werden. Es erscheint weiter wichtig, ein geeignetes Kulturmedium zu wählen, um die Raten der Fertilisierung und Implantation zu verbessern. Bemerkenswert ist, da $\beta$ die Mißbildungsrate nach den derzeitig vorliegenden Befunden bei ICSI nicht höher liegen als bei klassischer IVF. Indikationen für ICSI sind die erfolglose klassische IVF, das schwere OAT-Syndrom, die ausgeprägte Teratozoospermie, akrosomale Defekte und die therapierefraktäre Verschlußazoospermie.

Abgeschlossen wurde der Kongreß mit einigen Anmerkungen zu ethischen Aspekten der assistierten Reproduktion und der Zukunft der Reproduktionsmedizin durch Bettendorf (Hamburg), Edwards (Cambridge) und te Velde (Utrecht). Kritische Einwürfe beschäftigen sich insbesondere damit, daß durch ICSI die natürlichen Selektionsprinzipien für „schlechte Spermatozoen“ ausgeschaltet werden. Darüber hinaus wird die Diagnostik und kausale Therapie des andrologischen Sterilitätsfaktors in ihrer Wertigkeit weiter vermindert, da keine Notwendigkeit mehr besteht, derartige Defekte aufzudecken. Die Langzeitauswirkung von ICSI über Generationen sind bisher nicht bekannt. Te Velde (Utrecht) meinte, daß die Reproduktionsmedizin durch ihre sich immer verfeinernde Technik zum Selbstzweck werden könnte. Er warnte insbesondere vor der Gefahr, potentiell fertilen Paaren reproduktionsmedizinisch zu Kindern zu verhelfen. Nach seinen Erfahrungen treten bei Paaren, die nach dem ersten Jahr keine Schwangerschaft erreicht haben, innerhalb des nächsten Jahres noch in $\mathbf{5 4 \%}$ Schwangerschaften auf.

Zusammenfassend bot das Heidelberger Andrologie-Symposium einen hervorragenden Überblick über den aktuellen klinischen und wissenschaftlichen Stand der Reproduktionsmedizin mit einem guten Ausblick auf weitere Entwicklungsmöglichkeiten. Kritisch anmerken möchten die Verfasser des Berichtes, daß sich unseres Erachtens die Reproduktionsmedizin ohne ausreichende Kritik und Diskussion zu verselbständigen droht. Sie nutzt bisher nicht in ausreichendem Umfang ihre Chance, sich an der gesellschaftlichen Diskussion der sich wandelnden ethischen Normen, sterilen Paaren zu Kindern zu verhelfen, zu beteiligen.

\section{E. Ernst, Prof. Dr. W. Weidner}

Urologische Klinik der

Universitätsklinik Gießen

Klinikstraße 29

35385 Gießen 